\title{
A review of pleural infection in Northumbria Healthcare NHS Foundation Trust
}

\author{
Authors: Avinash Aujayeb, ${ }^{\mathrm{A}}$ Karl Jackson ${ }^{\mathrm{A}}$ and Kevin Conroy ${ }^{\mathrm{A}}$
}

\section{Introduction}

Understanding epidemiology of and deficiencies in care is crucial to inform practice. We serve 600,000 patients in a wellestablished pleural service. ${ }^{1}$

\section{Method}

A retrospective analysis of all patients with pleural infection between December 2016 and December 2017 was conducted.

\section{Results}

36 patients were identified, all being admitted from the community. The average age was 64.5 years and 19 were over 65 years of age.

Consolidation was present on chest X-ray in 24.

Comorbidities were malignancy (seven), alcohol excess (five), mental health (five), current smokers (nine) and ex-smokers (16). Drug use was recorded in one; eight had an HIV test.

Thoracic ultrasound findings were documented in 15 notes (commonest comment was 'multiloculated fluid' (11), others: 'small or moderate size' and 'echogenic').

28 samples of pleural fluid were available for analysis. Fluid was pus or turbid in 13, blood-stained in five, serous in six and four had no comments. $\mathrm{pH}$ result was available in 17 (and was $<7.2$ in 8); lactate dehydrogenase (LDH) was reported in 14 .

In 11 (39\%) samples cultures were positive: two Streptococcus pneumoniae, one Strep intermedius, one Actinomycosis turnicensis and Haemophilus parainfluenzae (in intravenous drug user), two Staphylococcus aureus (patients with indwelling pleural catheters). Other organisms included Strep dysgylactiae, Strep anginosus and mixed anaerobes.

Twenty-six had chest drains inserted with 10 receiving intrapleural lysis; nine received antibiotics only.

Thirty-five patients received piperacillin - tazobactam or co-amoxiclav initially. Clindamycin was given in $60 \%$ of cases, even for fully penicillin-sensitive organisms. Antibiotic duration was between 2 and 8 weeks.

The mean length of stay was 9 weeks (ICQ $1-56)$. All survived to discharge in first admission; three (9\%) had died

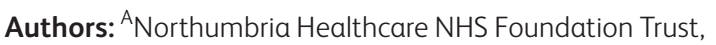
Newcastle upon Tyne, UK

\begin{tabular}{|l|l|l|}
\hline \multicolumn{3}{|c|}{ Pleural nltras ound or procedure } \\
Northumbria Healthcare NHS Foundation Trust
\end{tabular}

Fig 1. Pleural infection and procedure pro forma.

within 30 days, and three more within 6 months. Eight were readmitted within 38 days - 75\% due to infection - with $50 \%$ staying for 4 weeks.

\section{Conclusions}

Our data are in line with known epidemiology, microbiology, comorbidities and expected length of stay. ${ }^{2}$

We need to improve checking of HIV status, ultrasound reporting, sending for biochemical and microbiological analysis in accordance with British Thoracic Society guidance and in blood culture bottles, and to stop reliance on clindamycin. ${ }^{3-6}$

We have hence introduced a pleural infection and procedure pro forma (see Fig 1). 


\section{Avinash Aujayeb, Karl Jackson and Kevin Conroy}

\section{Conflicts of interest}

None declared.

\section{References}

1 Aujayeb A, Parker S, Bourke S, Miller J, Cooper D. A review of a pleural service. J R Coll Physicians Edinb 2016;46:26-31.

2 Bedawi EO, Hassan M, Rahman NM. Recent developments in the management of pleural infection: a comprehensive review. Clin Respir J 2008;12:2309-20.
3 HIV in Europe Secretariat. HIV indicator conditions: Guidance for implementing HIV testing in adults in health care settings. HIV in Europe Secretariat. www.eurotest.org/Portals/0/Guidance.pdf.pdf [Accessed 18 September 2019].

4 Evison M, Blyth KG, Bhatnagar R et al. Providing safe and effective pleural medicine services in the UK: an aspirational statement from UK pleural physicians. BMJ Open Respir Res 2018;5:e000307.

5 Davies CW, Gleeson FV, Davies RJ. BTS guidelines for the management of pleural infection. Thorax 2003;58(Suppl 2):ii18-28.

6 Menzies S, Rahman N, Wrightson J et al. Blood culture bottle culture of pleural fluid in pleural infection. Thorax 2011;66:658-62. 